\title{
A chemical sensor for alternative barriers using NIR spectroscopy
}

1 Jan C. Herzberg MSC

Trier University of Applied Sciences, Environmental Campus, Birkenfeld, Germany

2 Marinos Stylianou PhD

Subsurface Research Laboratory, Nireas International Water Research Center, University of Cyprus, Nicosia, Cyprus
3 Anahita Pourjabbar PhD

Subsurface Research Laboratory, Nireas International Water Research Center, University of Cyprus, Nicosia, Cyprus

4 Konstantinos Kostarelos PhD

University of Houston, Houston, TX, USA (corresponding author: kkostarelos@uh.edu)
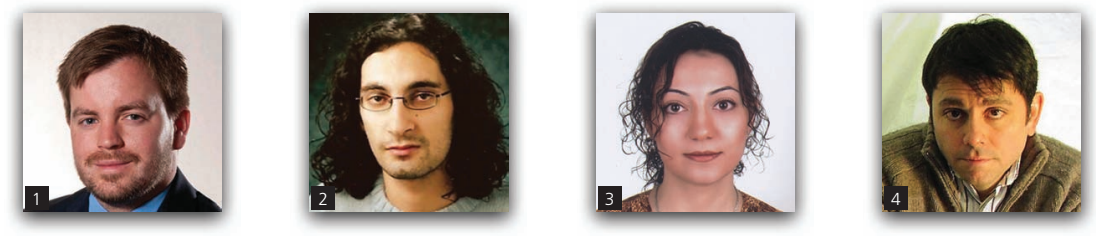

Remediation of contaminated sites often includes the use of alternative barriers as a passive treatment method for a variety of contaminants and sites. Alternative barriers, in turn, require a means for monitoring their performance directly, without the need for costly sampling. It is ideal that the monitoring system provide the data in real time and remotely. When combined with optical fibres, near-infrared (NIR) spectroscopy is a promising chemical sensor for in situ, on-site detection of contaminants. This technology has important advantages over other sensors: it can be used to identify hydrocarbons - capable of distinguishing between chemical groups such as alkanes and chlorinated, aromatic and polyaromatic hydrocarbons, as well as distinguishing compounds within the same homologous series of hydrocarbons. Furthermore, with the combined use of lipophilic polymer coatings and tip sensors, the waveguide can be turned into a localised chemical sensor. Coupled with the fact that the new generation of spectrometers are lower in cost and smaller in size than their predecessors, such a sensor can be installed in the field where communications technologies can transmit data from a remote location - ideally suited for alternative barriers as a means of monitoring the subsurface environment and the performance of the barrier.

\section{Introduction}

There is a need for a chemical sensor that can be used for environmental geotechnical applications; one that could be used for remote, on-site, long-term monitoring of soil and groundwater is ideal (Ghandehari et al., 2008). With this in mind, the focus of this research is the application of near-infrared (NIR) spectroscopy coupled with fibre optic waveguides to be used as a means of monitoring the subsurface environment and, hence, the performance of alternative barriers. Briefly, the advantages of the proposed technique include direct and rapid on-site measurements, remote operation capability and easy installation during barrier construction. In addition, the application of this sensor can be made for both permeable (reactive) and low-permeability alternative barriers.

The techniques currently used for on-site monitoring may be categorised and described as follows.

- Chromatographic techniques are highly accurate but relatively expensive. In addition, real-time measurements are difficult to acquire using chromatographic techniques; while an on-site laboratory can reduce the time between collection and analysis, samples are still analysed subsequent to collection and require on-site personnel.

- Electrochemical instruments are relatively inexpensive but are not capable of discriminating among species. Furthermore, the harsh subsurface environment lends to the degradation of electrical components, which precludes their use for longterm, on-site applications.

- Optical techniques are numerous. Laser-induced fluorescence (LIF), for example, has become one of the most established techniques for low-cost, on-site hydrocarbon monitoring. LIF geoprobes are available for the detection of polyaromatic hydrocarbon (PAH) contaminants (Baumann et al., 2000; Bulatov et al., 1997; Pepper et al., 2002; Quinn et al., 2002). The main drawback of LIF is that it only detects compounds that fluoresce (primarily PAHs), and it can be affected by the natural background fluorescence of humic substances; another shortcoming of LIF is the lack of species discrimination. 
Each of the three categories offers competing qualities such as selectivity, detection limit, robustness, low cost and ease of installation. The work presented in this paper was conducted for the development of an optical probe with a number of these qualities in mind, namely (a) wide-ranging detection capability, (b) low cost, (c) species discrimination and (d) long-term durability.

\section{Optical spectrometry}

Optical spectrometry is the study of the interaction between matter and radiated energy. It relies on the characteristic that every substance has a unique chemical structure. When photons permeate a material, a portion of the energy will be absorbed depending on the structure of the material. Due to the fact that each material is a unique combination of atoms, no two compounds produce the exact same electromagnetic radiation signature (Workaman and Weyer, 2007). Thus, the absorption spectra can be exploited in order to discriminate between compounds of interest. In some parts of this work, spectroscopy was used to identify unknown compounds using principal component analysis (PCA) (Lee et al., 2010; Raichlin and Katzir, 2008; Reboucas et al., 2011). However, when the compounds were of similar structure, this approach was shown to be difficult; Klavarioti et al. (2013) tried using PCA to identify alkanes by their spectral signature without success, and instead relied on other spectral characteristics.

\section{Infrared spectrometry}

The major difference between infrared (IR) spectrometry and other types of optical spectroscopy is in the frequency of absorbed electromagnetic energy: IR energy is in the range of the rotational levels of small molecules and of the vibration levels of molecular bonds. The frequencies and energies are characteristic of the respective compounds and are repeatable; thus, the spectrum of the absorbed energy can be relied on to identify materials that are being interrogated, used as a 'fingerprint' or 'signature' for a specific compound. As an example, the absorption signature of water is presented in Figure 1.

Others have investigated the use of optical sensors for use in the mid-infrared (MIR) region (Beyer et al., 2003; Karlowatz et al., 2004; Krska et al., 1993; McCue et al., 2006; Mizaikoff et al., 1995). A drawback of MIR probes is poor MIR energy transmission over long distances. NIR optical fibre sensors overcome this limitation to a large degree.

\section{Near-infrared spectrometry}

Researchers have investigated various aspects of the use of NIR spectroscopy for the on-site monitoring of the subsurface geo-environment (Albuquerque et al., 2005; Buerck et al., 2001; Conzen et al., 1993; Degrandpre and Burgess, 1990). One of the challenges of this application is the discrimination of the unknown contaminant(s) or the class of contaminant using a

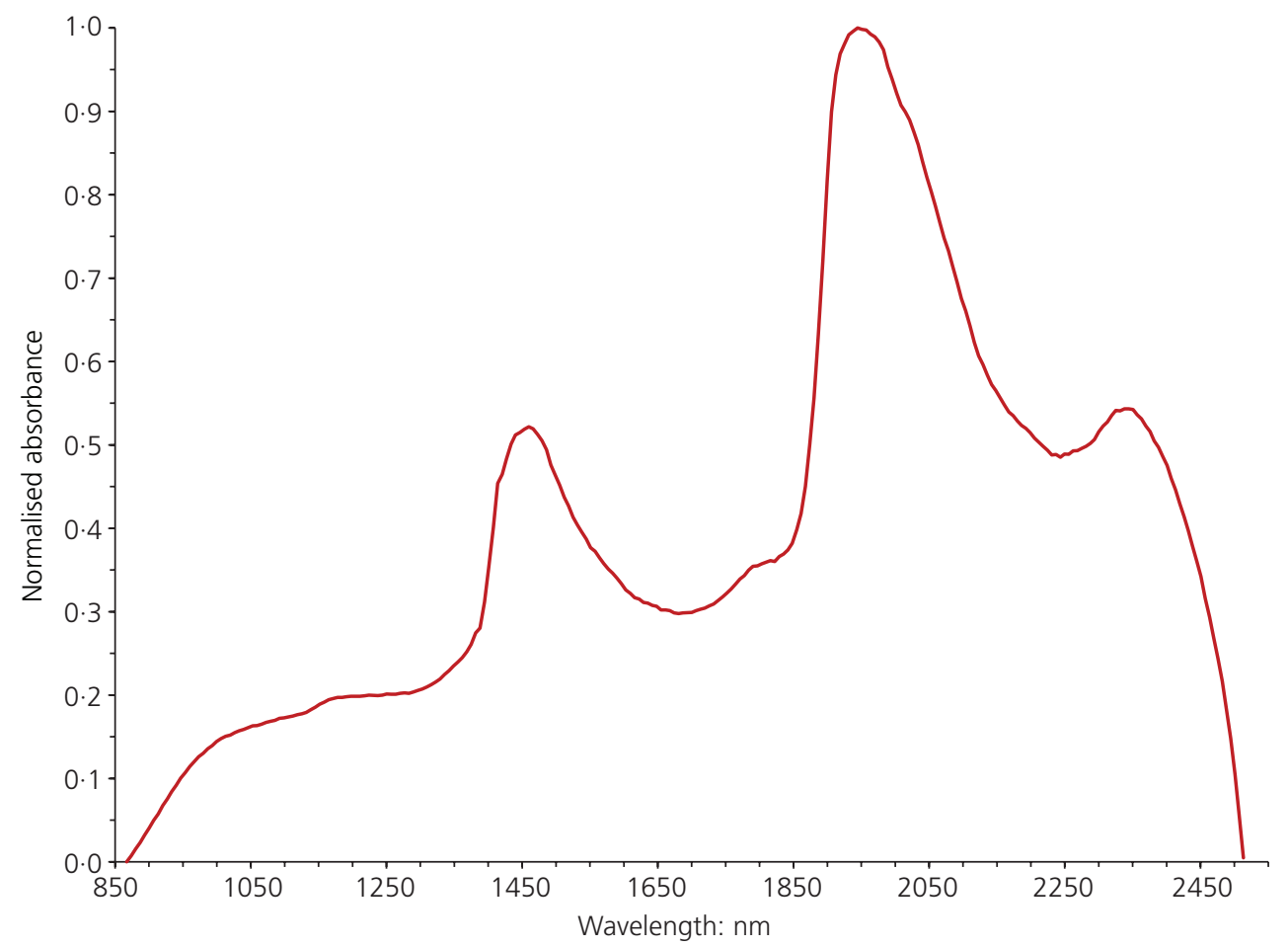

Figure 1. This signature spectrum of water was obtained using 50 measurements on individual drops and were each normalised and averaged 
spectral database. Ghandehari et al. (2008) and Klavarioti et al. (2013) reported the use of NIR absorption in order to identify and discriminate hydrocarbons from different classes as well as hydrocarbons from the same class for the purpose of real-time monitoring of the subsurface environment that relied on certain features of the NIR signature. It is important to note that not all the applications require that the chemical be identified but rather a means of identifying two phases (e.g. water against gasoline) is desired. Hence, based on the goal of the study, this method could be used for chemical mixtures for phase identification.

The peaks of the NIR absorption spectrum are the result of overtone and combination bands of fundamental molecular vibrations, in particular stretching and bending. Some of the specific molecular bonds which are very active in the NIR are listed below.

- $\mathrm{C}=\mathrm{O}$ is found in aldehydes, amides, carboxylic acids and esters.

- $\mathrm{C}-\mathrm{H}$ is found in aldehydes, alkanes, alkenes, alkynes and aromatic compounds.

- $\mathrm{C}-\mathrm{N}$ is found in amines, alkyl and aromatic compounds.

- $\mathrm{C}-\mathrm{O}$ is found in alcohols, ethers and esters.

- $\mathrm{O}-\mathrm{H}$ is found in alcohols and carboxylic acids.

\section{Advantages of NIR}

- There is a greater penetration depth when using NIR as compared to MIR light.

- Simple devices can be made by the use of quartz glass or sapphire.

- Measurements can be made within the permeable barrier (or immediately down-gradient) using optical fibre technology combined with NIR spectroscopy.

- Optical waveguides, components and accessories for NIR spectroscopy technique are comparatively low in cost.

\section{Disadvantages of NIR}

- The absorbance bands in the NIR are much wider than in the MIR and can overlap more.

- The technique has not been shown to be very sensitive for low aqueous concentrations (below $5000 \mathrm{mg} / \mathrm{l}$ ) at the present time, until researchers are able to develop hydrophobic coatings that will exclude water while allowing the organic compound to contact the waveguide - a promising approach that the authors have begun investigating.

- Water is a very strong absorber in the NIR range and this can interfere with the signal from other compounds in the case of aqueous solutions.

- Calibration is empirical in many cases, since there are no inverse models that can be used universally to relate the measured spectrum from an unknown to a database of spectra (Workaman and Weyer, 2007).

- The mirrors may undergo deposition and may need routine maintenance. Hence, the sensor may need to be accessible. Further durability testing will be needed.

\section{Experimental methods}

The experimental set-up consisted of three major elements: a light source (HL-2000-FHSA VIS-NIR, Ocean Optics), an NIR spectrometer (NIR Quest 256-2.5, Ocean Optics) and a bifurcated fibre optic probe (R600-7-VIS-125F, Ocean Optics). The sample drops are placed on a mirror for measurement so that the bifurcated fibre optical probe is directly above them (about $1.5 \mathrm{~cm}$ ). The end of the fibre optic probe is placed above the sample hydrocarbons that are on the mirror for measurement. NIR light is directed from the light source using an optical fibre and the bifurcated probe, through a drop of the hydrocarbon, reflected by the mirror back through the hydrocarbon, into the probe and finally to the spectrometer. By comparing the spectrum of the signal sent to the sample with the spectrum that returns, the energy absorbed by the liquid is measured. Additional details regarding the set-up and the measurements can be found in Klavarioti (2012) and Klavarioti et al. (2013).

The volume of each hydrocarbon drop that was placed on the mirror was kept small, in the range of $0 \cdot 2-0 \cdot 5 \mathrm{ml}$, in order to allow adequate time to complete the measurement. The variation in drop volume had an effect on the intensity of the adsorption spectrum, but not on the characteristic shape (i.e. location and shape of the peaks). This fact gave rise to the logic in the authors' handling of the data; the authors adjusted the baseline and then normalised each spectrum for a specific compound before averaging the spectra. Several spectra were obtained for each compound and were averaged to obtain the characteristic spectra or spectral absorbance signature for that compound.

Initially, a high number of measurements were made to ensure the repeatability of the experimental set-up; the spectrum of each alkane was measured 50 times in order to obtain the representative absorbance signature. Subsequently, and after statistical analysis of the initial results for the alkanes, only 20 measurements were necessary to obtain the spectra of binary and ternary hydrocarbon mixtures and the absorbance signatures for the petroleum fuels.

Since most non-aqueous phase liquids are a combination of several and often different hydrocarbons, and are not normally found in their pure form as environmental contaminants, the spectra of hydrocarbon mixtures were studied. The presumption was made that the spectrum for a mixture of two compounds should be comparable to the mathematical (calculated) addition of the absorbance signature for the two compounds alone. The assumption made is that the components of the mixture neither degrade nor react with each other, nor interfere with their movement - that is, vibration/rotation.

In order to validate this assumption, $5 \mathrm{ml}$ of heptane was mixed with

- $5 \mathrm{ml}$ of hexane (alkane)

- $5 \mathrm{ml}$ of dodecane (alkane) 
- $5 \mathrm{ml}$ of trichlorethylene (chlorinated hydrocarbon)

- $5 \mathrm{ml}$ of toluene (aromatic hydrocarbon)

a $5 \mathrm{ml}$ of toluene and $5 \mathrm{ml}$ of trichlorethylene.

An algorithm proposed by Klavarioti et al. (2013) was applied to the spectral signatures to determine the position of the peaks and compare the similarity of the two spectra. The approach taken also compares the relative heights of the peaks - that is, the peak-to-peak ratio. The algorithm was applied using MS Excel. As an additional check, measured spectra were compared to those published in the literature when possible (see Workaman and Weyer's (2007) appendix entitled 'Spectra-structure correlations for near infrared').

\section{Results and discussion}

Alkanes

The characteristic absorption signatures of six alkanes are presented in Figure 2, and it is noted that all alkanes exhibit four peaks: the most intense peak is between 1204 and $1210 \mathrm{~nm}$ (second overtone range), the second peak ranges between 1394 and $1407 \mathrm{~nm}$ (second combination band), the third peak lies between 1719 and $1732 \mathrm{~nm}$ (first overtone range), while the fourth peak is located between 2312 and $2319 \mathrm{~nm}$ (first combination band).
Although alkanes have similar absorption signatures, with the help of the algorithms that were applied, they can be distinguished from each other. The most important outcome is that all curves are distinctly different in comparison to the absorption signature of water (Figure 2), confirming the potential application of the technology as a chemical sensor in conjunction with alternative barriers. The absorption peaks are produced either by the overtone ranges of the methyl groups or by the combination bands. The first peak of the alkanes is called the second overtone range and is produced by the asymmetric vibration of the methyl group $\left(\mathrm{CH}_{3}\right)$. The wavelength of the second overtone range is increasing in direct relation to the length of hydrocarbon chain (Chung et al., 1999). The second peak is created by the second combination band; the third (first overtone range) and the fourth peaks (first combination band) are highly distinct, which makes them ideal for use in discriminating between the alkanes. Tosi and Pinto (1972) examined 50 linear and branched hydrocarbons, where all had five primary bands in the first overtone range, and they concluded that this peak was likely due to the asymmetric stretch of the methyl group. The first overtone range of a series of linear hydrocarbons yields the strongest peak (most intense absorption) of all overtones. The absorptivity of these combined peaks does not increase with the chain length because it has the contributions from two sources. The first combination band is rich in information and also very complex, as it includes combinations of $\mathrm{C}-\mathrm{H}$ stretching with

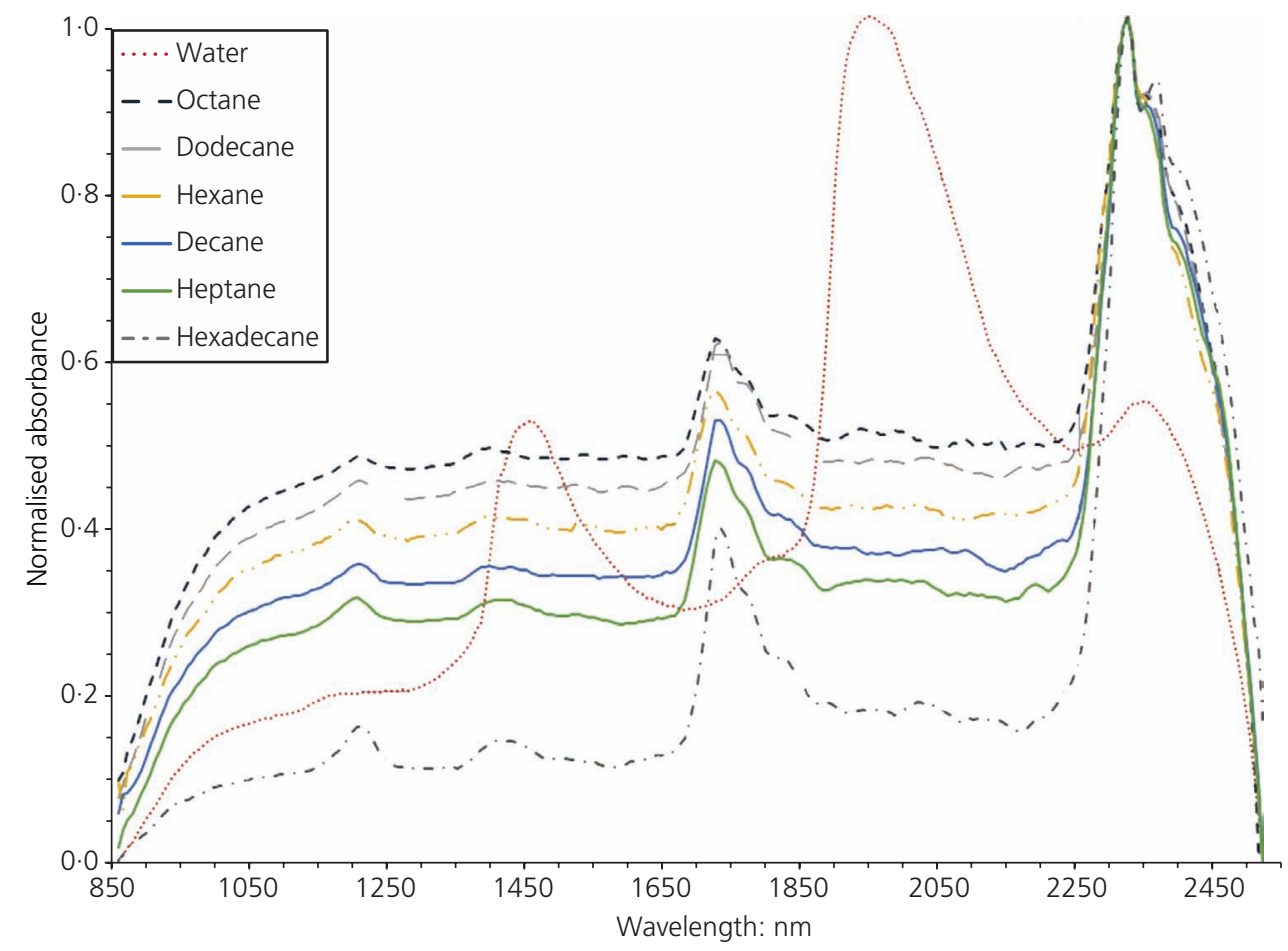

Figure 2. The signature for several alkanes have similarities, yet exhibit enough distinctness that can allow for their discrimination. The signature of water is easily identified in comparison to these alkanes 


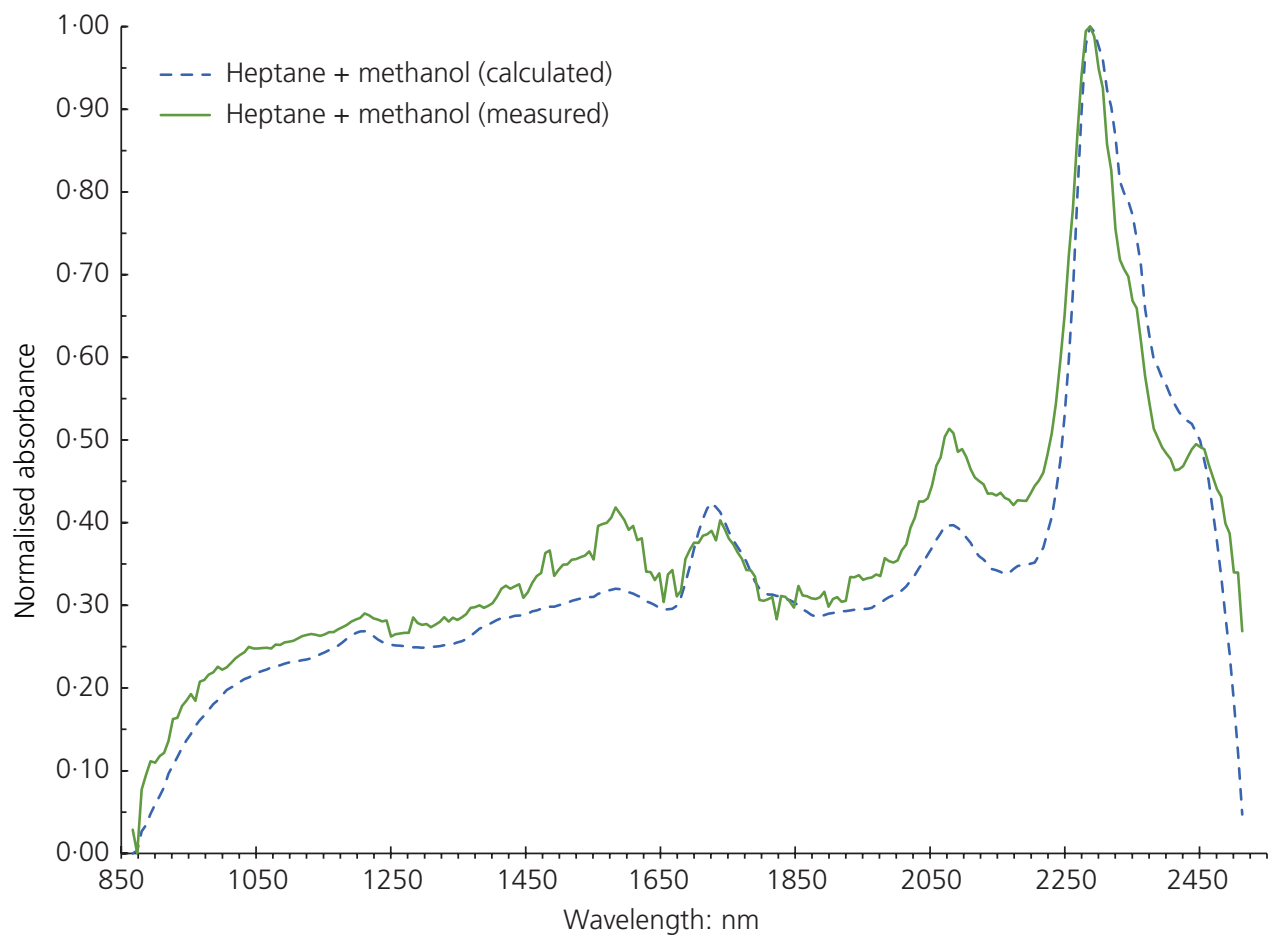

(a)

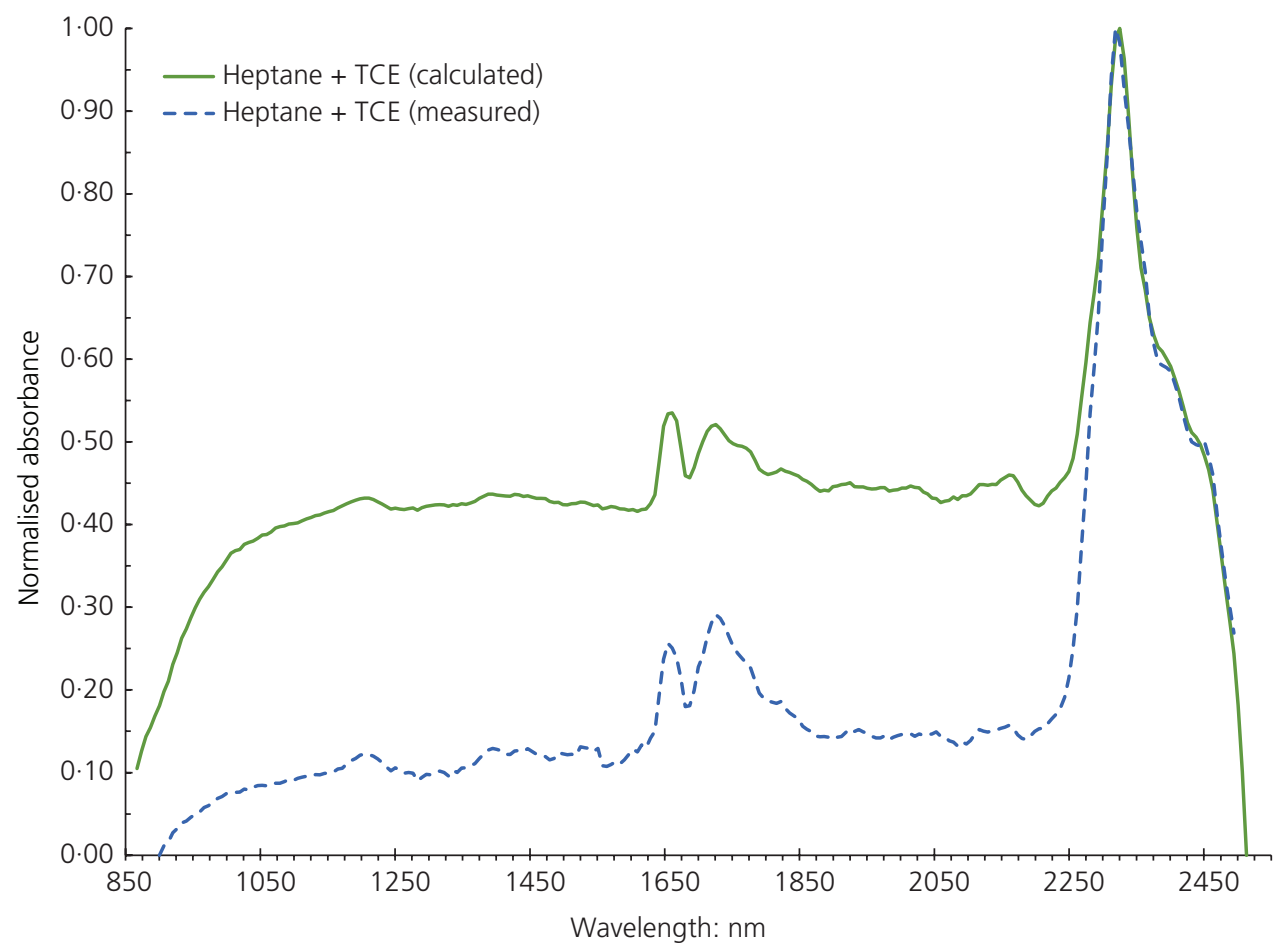

(b)

Figure 3. The signature of hydrocarbon mixtures are presented along with the spectra predicted from linear superposition of the spectra for the components. The binary and ternary mixtures were all equal parts of their respective components. (a) Comparison of the signature for a mixture of heptane and methanol with the calculated spectrum; (b) heptane with trichloroethylene (TCE); (c) heptane with hexane; (d) heptane with toluene; (e) heptane with dodecane; (f) heptane with toluene and TCE (continued on next page) 


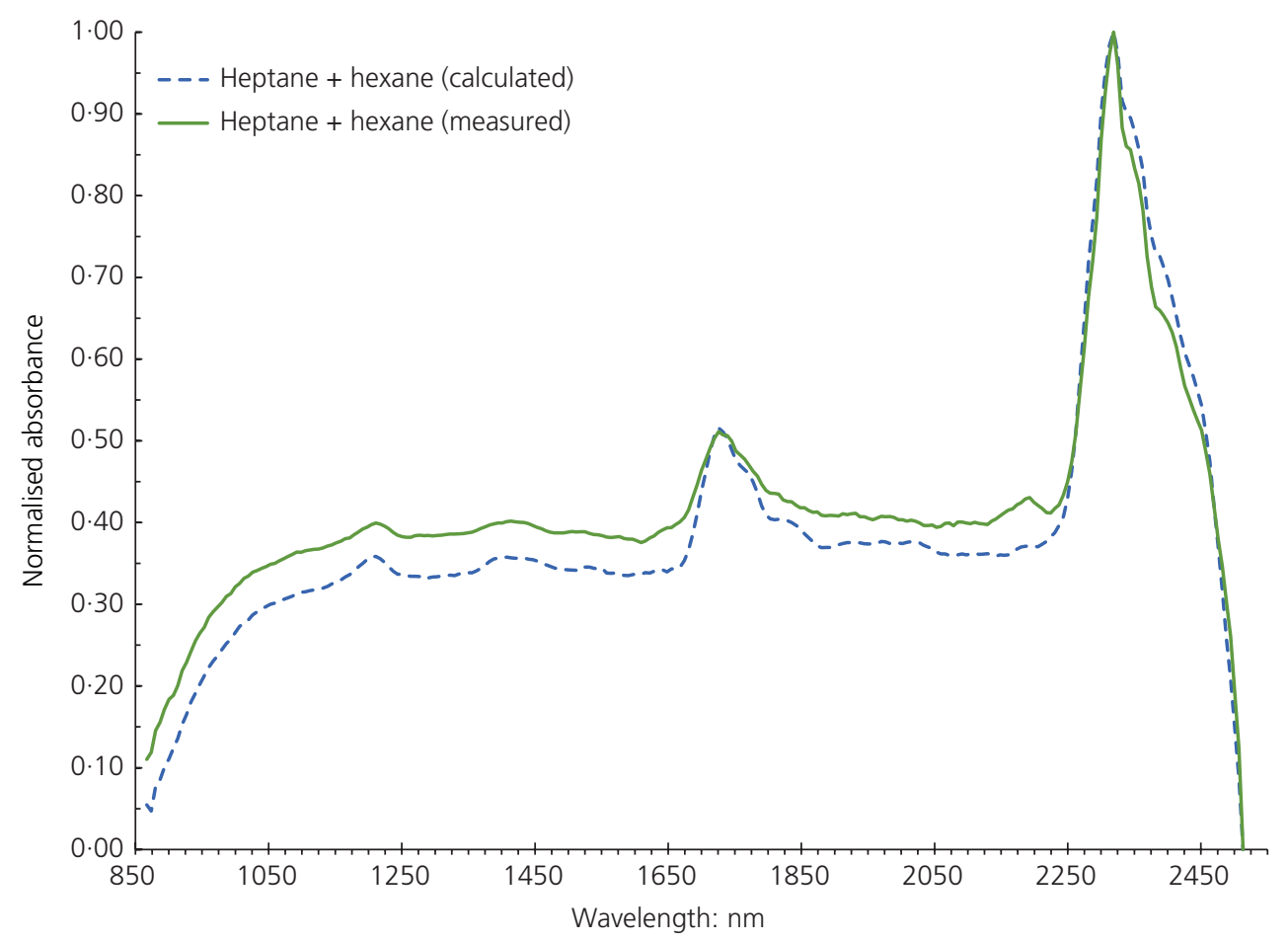

(c)

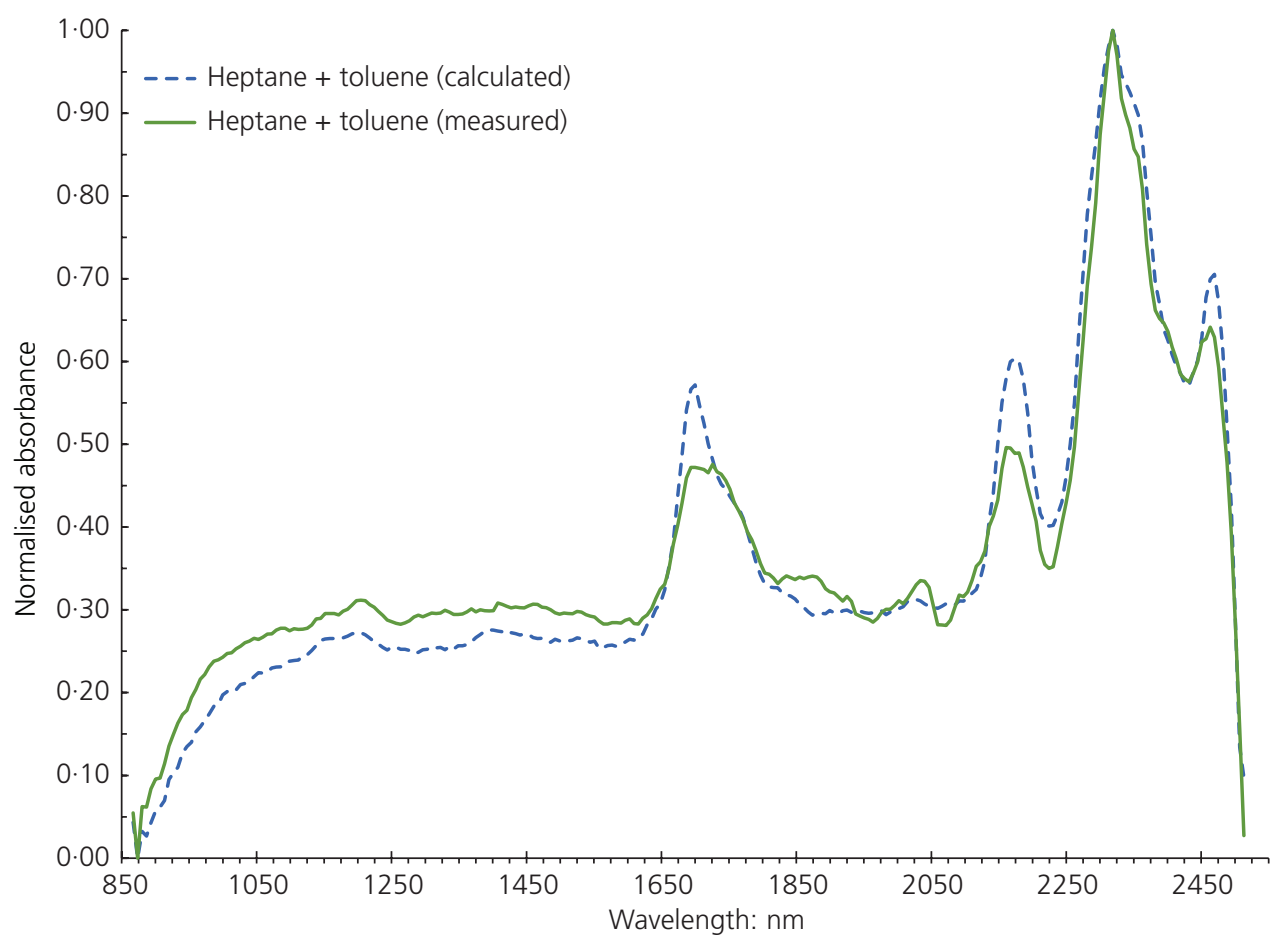

(d)

Figure 3. Continued 


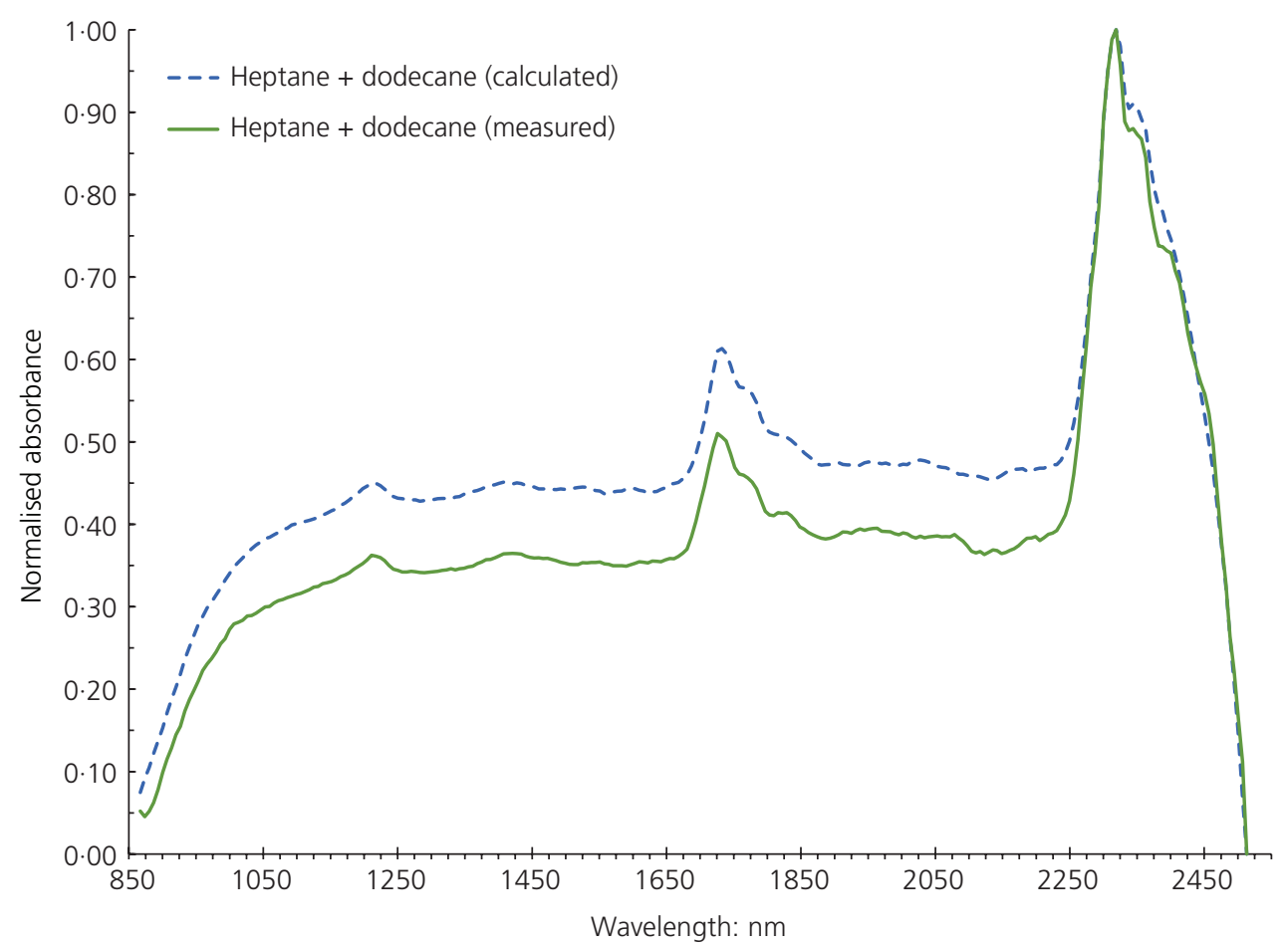

(e)

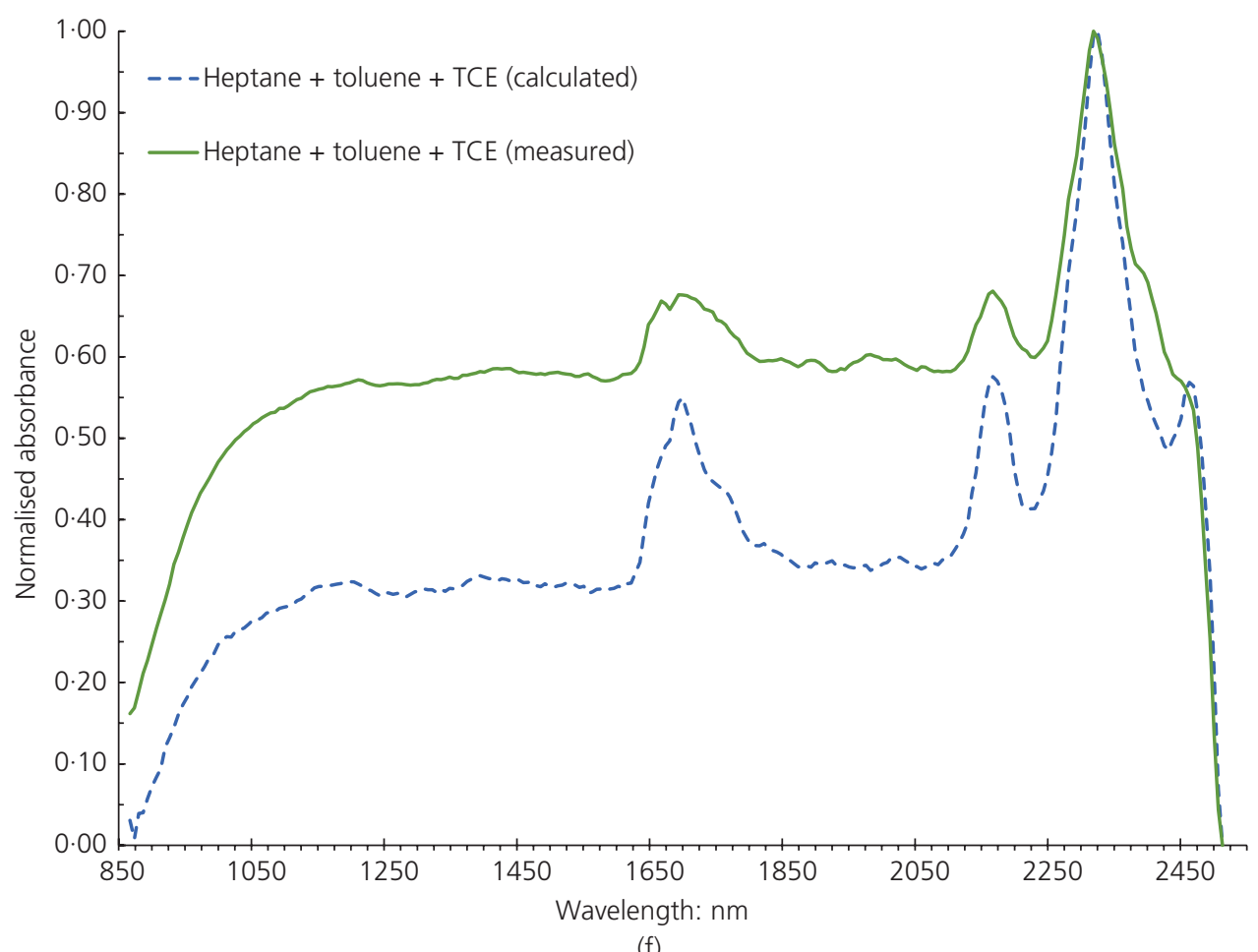

Figure 3. Continued 
A chemical sensor for alternative barriers

using NIR spectroscopy

Herzberg, Stylianou, Pourjabbar and Kostarelos

\begin{tabular}{lc}
\hline Mixture & Spectra \\
\hline Heptane/hexane & $99 \cdot 98 \%$ \\
Heptane/dodecane & $99 \cdot 99 \%$ \\
Heptane/trichlorethylene & $99 \cdot 83 \%$ \\
Heptane/toluene & $97 \cdot 72 \%$ \\
Heptane/methanol & $99 \cdot 98 \%$ \\
Heptane/trichlorethylene/toluene & $99 \cdot 86 \%$
\end{tabular}

Table 1. Statistical similarity between the calculated and measured spectra of binary and ternary mixtures using Student's t-test

various bending and also stretching vibrations from the MIR region as well (Workaman and Weyer, 2007).

\section{Mixtures of hydrocarbons}

With respect to the binary hydrocarbon mixtures (see Figure 3), the peaks observed in the NIR absorption spectrum for the mixture correspond with the signatures for the individual components. Furthermore, the results show that the spectrum for the mixture can be calculated mathematically as the sum (linear superposition) of the two individual absorption signatures for those that were tested.
In order to compare the similarity between the calculated and measured absorption spectra, Student's $t$-test was performed. Visually, one cannot easily judge a degree of similarity and it leads to a subjection judgement; this is why the statistical analysis is applied in order to qualify the similarity. The statistical similarity between the calculated and the measured absorption spectrum was high for the five binary mixtures and one ternary mixture, as shown in Table 1.

By comparing the measured spectra to the calculated spectra qualitatively, it appears that as the number of components in the mixture increases, the molecular vibration is inhibited. Note that the intensity of the second overtone range and the second combination band in Figure 3 are very weak and are hardly detectable. To be more specific, the ratio of absorption intensity of the second overtone range to the first overtone range is 1:10 for the pure heptane, but in the binary mixtures, the intensity of the second overtone almost disappears and is no longer detected by the algorithm. The same can be said for the first overtone range and the first combination band.

\section{Petroleum fuels}

All the petroleum fuels have spectra that are significantly different from that of water, indicating the potential application of this technology within the subsurface environment as a chemical sensor (see Figure 4). Examination of the spectra reveals

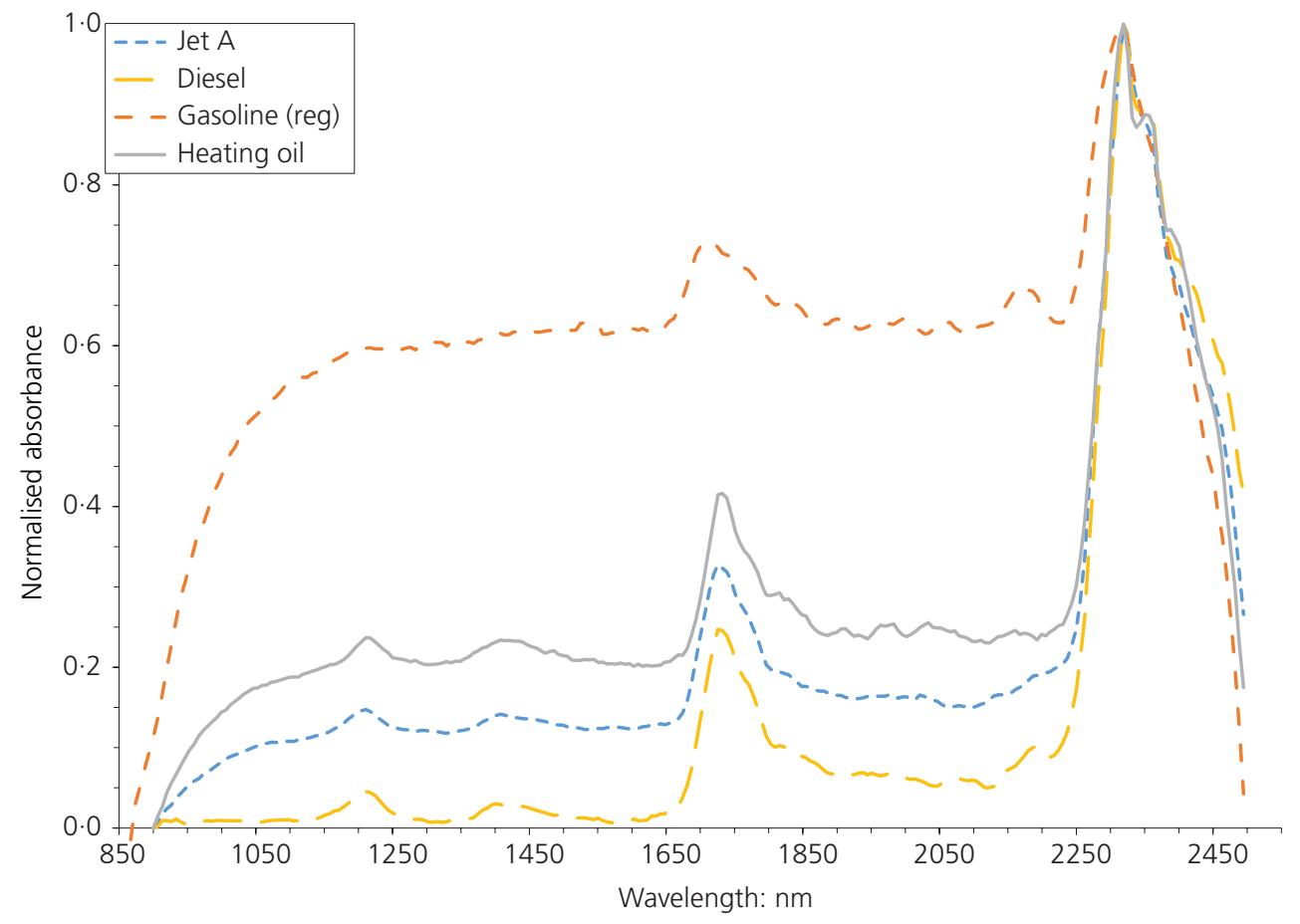

Figure 4. Signatures of several common fuels were obtained. As in the case of the alkanes, although the signatures presented here appear to have similarities, there are distinctions that can be relied on to discriminate between them provided that the fuels themselves are manufactured consistently 
considerable spectral differences between the petroleum products as well. Although the petroleum fuels are mixtures of numerous hydrocarbons, because they are manufactured and blended with a high degree of consistency, the spectra for a particular fuel can be considered as an absorbance signature useful for discrimination. The main constituents of petroleum fuels are alkanes, and this can be easily recognised while comparing the spectra in Figure 4 to those in Figure 2. Furthermore, the spectra were compared with absorption signatures in the authors' database in order to discriminate the constituents of the fuel. Table 2 presents the results of this comparison, where the hydrocarbons that were identified in the petroleum spectra are indicated with an X, while those not present (based on spectroscopic results) are indicated with an $\mathrm{O}$.

Toluene, as a representative aromatic hydrocarbon, can be distinguished by its $\mathrm{C}-\mathrm{H}$ aryl groups. The small peaks in the first combination band of the spectra for gasoline $(2167 \mathrm{~nm})$ and diesel $(2186 \mathrm{~nm})$ are indications of $\mathrm{C}-\mathrm{H}$ aryl groups, thus indicating the presence of toluene in these two fuels. Gasoline and diesel, indeed, contain a high concentration of aromatic hydrocarbons. Therefore, the spectral features of gasoline and diesel are significantly different from those of other petroleum products, such as the aromatic $\mathrm{C}-\mathrm{H}$ bond at 1217 and $1706 \mathrm{~nm}$ (Chung et al., 1999).

The most useful spectral information is located between 1204 and $1407 \mathrm{~nm}$ and between 1706 and $2319 \mathrm{~nm}$. The spectral differences are more prominent in the $1899-2319 \mathrm{~nm}$ (first combination band) range compared to those of the 1204-1217 nm (second overtone range) range. Since the first combination band is very erratic, it is very difficult to use it as a means of discriminating between the fuels. As the second overtone range is a function of the $\mathrm{CH}_{2} / \mathrm{CH}_{3}$ ratio in linear hydrocarbons, it can be used to identify the length of the hydrocarbon chain: as the number of $\mathrm{CH}_{2}$ groups relative to the $\mathrm{CH}_{3}$ groups increases, the location (wavelength) of the second overtone also increases (Chung et al., 1999). After considering this and examining the measured spectra, gasoline appears to have the longest

\section{Alkanes}

Aromatic hydrocarbons (C-H aryl groups)

\begin{tabular}{lll}
\hline Diesel & $X$ & $X$ \\
Gasoline & $X$ & $X$ \\
Jet fuel & $X$ & 0 \\
Heat oil & $X$ & 0
\end{tabular}

Table 2. Identification of compounds within fuels using their spectra and those of the database (hydrocarbons identified in the petroleum spectra are indicated with an $\mathrm{X}$, while those not identified are indicated with an O) chain length (second overtone range at $1217 \mathrm{~nm}$ ), while Jet A fuel has the shortest chain length (second overtone range at $1204 \mathrm{~nm})$.

Of all the tested materials, gasoline has the widest variety of compounds and because of this, the peaks are very small in intensity but broad. In the study of mixtures (section titled 'Mixtures of hydrocarbons'), it was noted that increasing the number of components tends to inhibit molecular vibration, which in turn diminishes the intensity of the absorption spectrum for the mixture. Overall, however, enough unique spectral features of each petroleum product exist to provide a basis for the qualitative discrimination of each. It is expected that there will be spectral overlaps between products that have highly similar physical properties.

\section{Dissolved elements}

A preliminary study of the spectra of aqueous solutions of several dissolved elements $(1000 \mathrm{mg} / \mathrm{l}$ dissolved in a $0.5 \mathrm{M}$ solution of nitric acid $\left(\mathrm{HNO}_{3}\right)$ ) was performed, with the intention of identifying unknown heavy metals and contaminants in groundwater by comparing their spectra to the absorption signatures (see Figure 5). These spectra were measured only five times (each) in order to get a first look at their spectra in order to understand whether the NIR approach could be used for the intended purpose. After applying the algorithms to locate the peaks, it was noted that all measured elements have three significant peaks as does water at $1459 \mathrm{~nm}$ (first overtone range), at $1944 \mathrm{~nm}$ (first combination band) and between 2312 and $2344 \mathrm{~nm}$ (first combination band).

It is clear that the absorption spectra of these dissolved elements (arsenic, cadmium, cobalt, copper, chromium, iron, lead, manganese, nickel and zinc) are nearly the same as that of water (also shown in Figure 5), an indication that the water spectrum is dominant with respect to the elements. Student's $t$-test confirmed that the similarity between the absorption signature of water and the spectra of the dissolved elements is higher than $80 \%$. The minima/maxima and the points of inflection arising from the water are too intense, making it difficult to discriminate between the elements in the aqueous environment using these criteria alone.

The $\mathrm{O}-\mathrm{H}$ vibration of water in both cases is very active in the NIR range and the absorbed energy is too intense to detect the elements within the water structure. In theory, the elements are present in water in the form of ions and they can affect the NIR spectra. The effects of specific ions differ, depending on their size and electronic characteristics. These effects can be

- charge-dipole interactions between the ions and the water molecules that affect the hydrogen bonding of the water itself

- the formation of hydrogen bonds between an oxygen or a nitrogen in the ions and water

- the presence of $\mathrm{OH}$ itself or $\mathrm{OH}$ within the ion (Workaman and Weyer, 2007). 


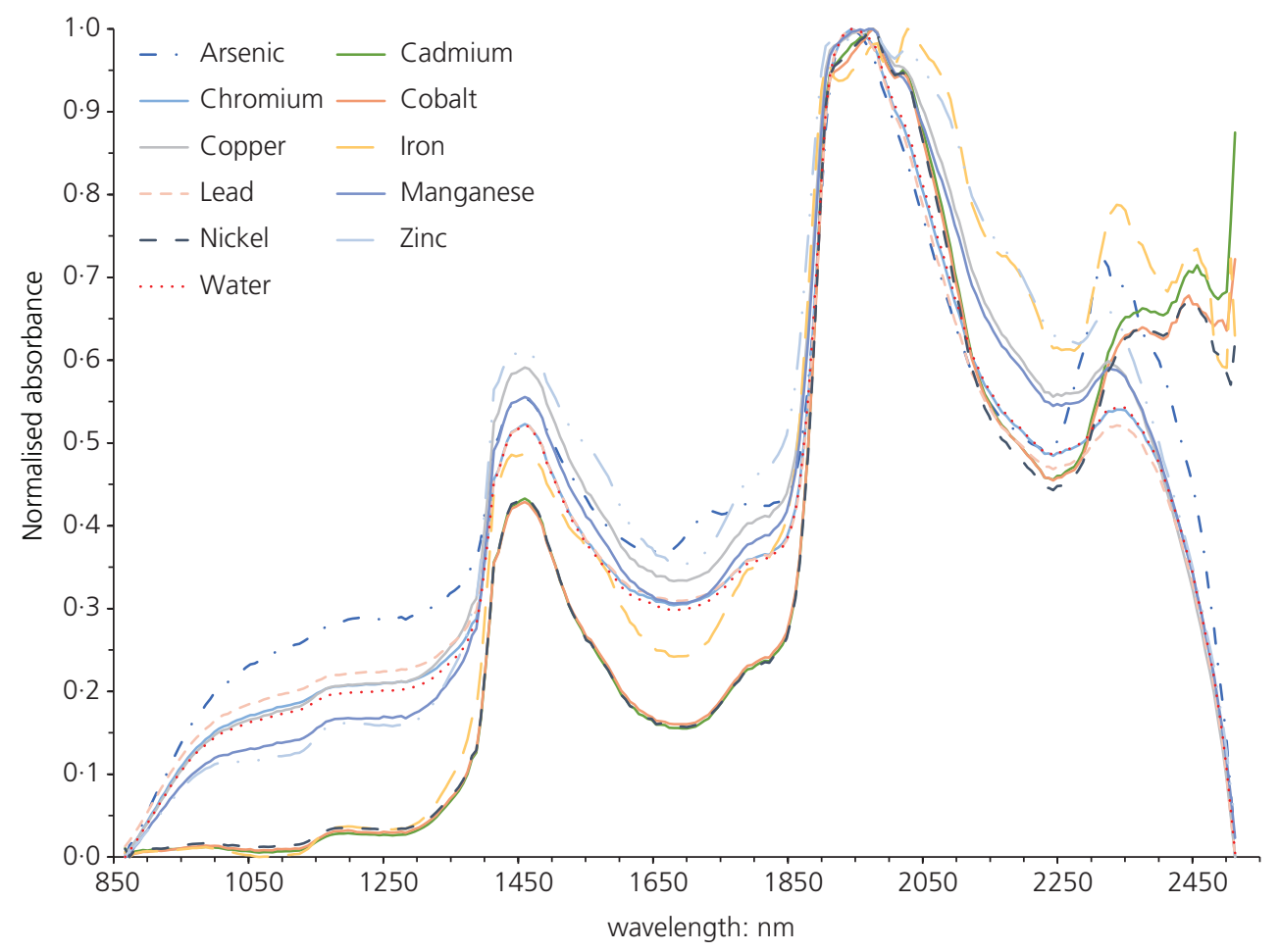

Figure 5. Preliminary studies involving elements are presented here. The elements are all dissolved in aqueous solutions, and it is apparent that their spectra are significantly masked by the strong signal from the water, also shown. Although some distinctness in the signatures can be noticed, it is clear that a method to eliminate the water signal is needed
It will be difficult to identify heavy metals and other elements dissolved in water at low concentrations using the algorithms alone, as originally proposed. The measurements validate the disadvantage previously mentioned that NIR spectroscopy is not very sensitive for low concentrations. The low concentration of the elements $(1000 \mathrm{mg} / \mathrm{kg})$ in the samples that were prepared and measured is likely the reason for the difficulty in their detection. According to Shi et al. (2014), heavy metals should have a concentration of at least $4000 \mathrm{mg} / \mathrm{kg}$. Concentrations on that order occur in mining or industrial areas, so that the sensor could be useful in such locations. In most areas, heavy metal concentrations in the subsurface are usually found at trace levels or lower (Shi et al., 2014). Nonetheless, a careful examination of the spectra indicates that there are subtle differences between the spectra for the dissolved elements which may be exploited for identification purposes. Further work will be needed to develop a method to exclude water molecules during the sensor operation (e.g. hydrophobic coating), or a methodology/algorithm for this goal.

\section{Conclusions and practical implications}

It is certain, based on these preliminary results, that the sensor could be used for the detection of hydrocarbons. The results for alkanes, chlorinated hydrocarbons, aromatic hydrocarbons and various fuels were very promising, but they will require additional research. All the petroleum fuels have an absorption signature different from that of water, indicating that the presence of a fuel within the subsurface environment can be detected. Hence, this method is useful when the application requires the detection of a phase, for example water against gasoline, as would be desired in the area surrounding an underground tank.

Furthermore, it is possible to distinguish between petroleum types and to detect the major compounds and some traces of other ingredients in the fuels. Additional studies with a full range of commercially available fuels are necessary to confirm that discrimination based on the absorption signature of fuels will be possible for all types of petroleum fuels.

Further studies are also recommended with regard to the other classes of hydrocarbons. While the results presented here are encouraging, a database of all the environmental contaminants most commonly found in the subsurface needs to be developed. Finally, the methodology for resolving the spectra of hydrocarbon mixtures into the individual components needs to be developed further. The results here are promising and a strategy that includes various coatings, each designed for specific hydrocarbon classes or to exclude water from the measurement, could be useful in that regard. The use of coatings has been shown to be useful when the sensor is to be used for the detection of organic compounds in aqueous solutions (Ghandehari et al., 2008). The authors believe that, based on the preliminary studies of several elements, a hydrophobic 
Environmental Geotechnics

Volume 4 Issue EG4
A chemical sensor for alternative barriers

using NIR spectroscopy

Herzberg, Stylianou, Pourjabbar and Kostarelos coating could also be employed when the detection of dissolved elements is desired and it will lower the sensitivity of detection.

In summary, the ambition of a monitoring system within the subsurface environment that can be used in real time can be fulfilled with an NIR-based chemical sensor. A monitoring system employing an NIR waveguide has positive characteristics which are ideal for geo-environmental applications such as alternative barriers. Optical wave-guides are not expensive, easy to operate/install and very robust and can be used in harsh environments. These advantages, combined with the advantages of NIR spectroscopy (e.g. the higher penetration depth of the electromagnetic radiation and the high activity for $\mathrm{C}-\mathrm{H}$ bonds), make them ideal sensors for hydrocarbons in the ecosystem.

\section{Acknowledgements}

The authors would like to acknowledge the support of the Nireas International Water Research Center (NEA IPODOMI/STRATH II/0308/09) co-funded by the Republic of Cyprus and the European Regional Development Fund, the University of Cyprus (CY) and Erasmus Student Exchange Program (EU), which collectively funded this project.

\section{REFERENCES}

Albuquerque JS, Pimentel FM, Valdinete LS et al. (2005) Silicone sensing phase for detection of aromatic hydrocarbons in water employing near-infrared spectroscopy. Analytical Chemistry 77(1): 72-77, http://dx.doi.org/10.1021/ac0495788.

Baumann T, Haaszio S and Niessner R (2000) Applications of a laser-induced fluorescence spectroscopy sensor in aquatic systems. Water Research 34(4): 1318-1326, http://dx.doi.org/ 10.1016/S0043-1354(99)00260-2.

Beyer T, Hahn P, Hartwig S et al. (2003) Mini spectrometer with silver halide sensor fiber for in situ detection of chlorinated hydrocarbons. Sensors and Actuators B: Chemical 90(1-3): 319-323, http://dx.doi.org/10.1016/S0925-4005(03)00054-6.

Buerck J, Roth S, Kraemer K, Scholz M and Klaas N (2001) Application of a fiber-optic NIR-EFA sensor system for in situ monitoring of aromatic hydrocarbons in contaminated groundwater. Journal of Hazardous Materials 83(1-2): 11-28, http://dx.doi.org/10.1016/S0304-3894(00)00335-6.

Bulatov V, Gridin VV, Polyak F and Schechter I (1997) Application of pulsed laser methods to in-situ probing of highway originated pollutants. Analytica Chimica Acta 343(1-2): 93-99, http://dx.doi.org/10.1016/S0003-2670(97)00026-3.

Chung H, Choi HJ and Ku MS (1999) Rapid identification of petroleum products. Bulletin of the Korean Chemical Society 20(9): 1021-1025.

Conzen JP, Burck J and Ache HJ (1993) Characterization of a fiber-optic evanescent wave absorbance sensor for nonpolar organic compounds. Applied Spectroscopy 47(6): 753-763, http://dx.doi.org/10.1366/0003702934066956.

Degrandpre MD and Burgess LW (1990) A fiber-optic FT-NIR evanescent field absorbance sensor. Applied Spectroscopy 44(2): 273-279, http://dx.doi.org/10.1366/0003702904085453.
Ghandehari M, Kostarelos K, Cheng KC, Vimer A and Yoon S (2008) Near-infrared spectroscopy for in-situ monitoring of geoenvironment. ASCE Journal of Geotechnical and Geoenvironmental Engineering 134(4): 487-496, http://dx.doi.org/10.1061/(ASCE)1090-0241(2008)134:4(487).

Karlowatz M, Kraft M and Mizaikoff B (2004) Simultaneous quantitative determination of benzene, toluene, and xylenes in water using mid-infrared evanescent field spectroscopy. Analytical Chemistry 76(9): 2643-2648, http://dx.doi.org/ 10.1021/ac0347009.

Klavarioti M (2012) A Sensor Utilizing Near Infrared Spectroscopy for Monitoring of the Geo-environment. MSc thesis, University of Cyprus, Nicosia, Cyprus.

Klavarioti M, Kostarelos K, Pourjabbar A and Ghandehari M (2013) In situ sensing of subsurface contamination: Part I: Near-infrared spectral characterization of alkanes, aromatics, and chlorinated hydrocarbons. Environmental Science and Pollution Research 21(9): 5849-5860, http://dx.doi.org/ 10.1007/s11356-013-2478-z.

Krska R, Taga K and Kellner R (1993) New IR fiber-optic chemical sensor for in situ measurements of chlorinated hydrocarbons in water. Applied Spectroscopy 47(9): 1484-1487.

Lee WS, Alchanatis V, Yang C et al. (2010) Sensing technologies for precision specialty crop production. Computers and Electronics in Agriculture 74: 2-33, http://dx.doi.org/10.1016/ j.compag.2010.08.005.

McCue RP, Walsh JE, Walsh F and Regan F (2006) Modular fibre optic sensor for the detection of hydrocarbons in water. Sensors and Actuators B: Chemical 114(1): 438-444, http://dx.doi.org/10.1016/j.snb.2005.04.048.

Mizaikoff B, Taga K and Kellner R (1995) Infrared fiber optic gas sensor for chlorofluorohydrocarbons. Vibrational Spectroscopy 8(2): 103-108, http://dx.doi.org/10.1016/09242031(94)00077-T.

Pepper JW, Wright AO and Kenny JE (2002) In-situ measurements of subsurface contaminants with a multichannel laser-induced fluorescence system. Spectrochimica Acta Part A: Molecular and Biomolecular Spectroscopy 58(2): 317-331.

Quinn MF, Alemeddine O, Al-Awadi E et al. (2002) The application of laser-induced fluorescence techniques for the measurement of hydrocarbons in the groundwater of Kuwait. Instrumentation Science \& Technology 30(1): 79-95, http://dx.doi.org/10.1081/CI-100108773.

Raichlin Y and Katzir A (2008) Fiber-optic evanescent wave spectroscopy in the middle infrared. Applied Spectroscopy 62(2): 55A-72A, http://dx.doi.org/10.1366/ 000370208783575456.

Reboucas MV, Brandão DS, Trindade A, Pimentel MF and Teixeira LSG (2011) Chemical composition determination of complex organic-aqueous mixtures of alcohols, acetone, acetonitrile, hydrocarbons and water by near-infrared spectroscopy. Vibrational Spectroscopy 55(2): 172-182, http://dx.doi.org/10.1016/j.vibspec.2010.11.003. 
Shi T, Chen Y, Lieu Y and Wu G (2014) Visible and near-infrared reflectance spectroscopy: an alternative for monitoring soil contamination by heavy metals. Journal of Hazardous Materials 265: 166-176, http://dx.doi.org/10.1016/j.jhazmat. 2013.11.059.
Tosi C and Pinto A (1972) Near-infrared spectroscopy of hydrocarbon functional groups. Spectrochimica Acta 28A(23): 585-597, http://dx.doi.org/10.1016/0584-8539(72)80243-5.

Workaman J and Weyer L (2007) Practical Guide to Interpretive Near-Infrared Spectroscopy. CRC Press, London, UK.

\section{HOW CAN YOU CONTRIBUTE?}

To discuss this paper, please submit up to 500 words to the editor at journals@ice.org.uk. Your contribution will be forwarded to the author(s) for a reply and, if considered appropriate by the editorial board, it will be published as a discussion in a future issue of the journal. 